\title{
Baroclinic generation of potential vorticity in an embedded planet-disk system
}

\author{
Jianghui $\mathrm{JI}^{1}$, Shangli $\mathrm{OU}^{2}$ and Lin LIU $^{3}$ \\ ${ }^{1}$ Purple Mountain Observatory, Chinese Academy of Sciences, Nanjing 210008, China \\ email: jijh@pmo.ac.cn \\ ${ }^{2}$ High Performance Computing, Center for Computation and Technology/Information \\ Technology Services, Louisiana State University, Baton Rouge, LA 70803 \\ email: ou@cct.lsu.edu \\ ${ }^{3}$ Department of Astronomy, Nanjing University, Nanjing 210093, China
}

\begin{abstract}
We use a multi-dimensional hydrodynamics code to study the gravitational interaction between an embedded planet and a protoplanetary disk with emphasis on the generation of vortensity (Potential Vorticity or PV) through a Baroclinic Instability. We show that the generation of $\mathrm{PV}$ is very common and effective in non-barotropic disks through the Baroclinic Instability, especially within the coorbital region. Our results also complement previous work that non-axisymmetric Rossby-Wave Instabilities (RWIs) are likely to develop at local minima of PV distribution that are generated by the interaction between a planet and an inviscid barotropic disk. The development of RWIs results in non-axisymmetric density blobs, which exert stronger torques onto the planet when they move to the vicinity of the planet. Hence, large amplitude oscillations are introduced to the time behavior of the total torque acted on the planet by the disk. In current simulations, RWIs do not change the overall picture of inward orbital migration but cause a non-monotonic behavior to the migration speed. As a side effect, RWIs also introduce interesting structures into the disk. These structures may help the formation of Earth-like planets in the Habitable Zone or Hot Earths interior to a close-in giant planet.
\end{abstract}

Keywords. accretion, accretion disks - Baroclinic Instability - Rossby-wave instability - hydrodynamics - numerical methods - planetary systems: protoplanetary disks

\section{Introduction}

The standard core-accretion theory (Safronov 1969; Lissauer 1993) suggests that the formation of planets in circumstellar disks around $\mathrm{T}$ Tauri stars consists of the formation of planetesimals via collisions/coalitions of dust grains in the early stage and then gravitationally accretion after they accumulate enough mass. The lifetime of this T Tauri star phase is estimated to be short ( $\lesssim 10^{7}$ years). In order for cores of protoplanets with Jupiter mass to accumulate enough material in the T Tauri stage, it is thought that their cores have to form outside the so-called "ice line" located far away from the central star (typically beyond $\sim 4 \mathrm{AU}$, see Ida \& Lin 2004) so that the temperature is low enough to allow the condensation of gas materials to solid ice. These additional solid grains helps to increase the dust coagulation speed and shorten the time needed to form the embryos of protoplanets. From the observational side, recent discoveries of extrasolar planets show that a large number of the host stars are surrounded by "hot Jupiters" and close-in super Earths. Around $80 \%$ of the extrasolar planets are in orbits with semi-major axes in the range $0.01 \lesssim a \lesssim 2.5 \mathrm{AU}$, and $\sim 25 \%$ of the total population are short-period planets with $a \lesssim 0.1$ AU. This has brought one of the most interesting puzzles to theorists: if protoplanets had formed in a disk region beyond $\sim 4 \mathrm{AU}$ from the central star, how did the observed extrasolar planets end up with orbits that are so close to their host stars? If 
the standard theory for the formation of protoplanetary cores holds (Pollack et al. 1996; Ida \& Lin 2004), then the giant planets (like hot-Jupiters) must have undergone inward orbital migration to their current locations.

Goldreich \& Tremaine (1978) and many other authors studied the gravitational interaction between a gaseous protoplanetary disk and an embedded planet (or satellite). The planet causes the formation of spiral waves inside the disk at the Lindblad resonances; as a result of the density asymmetry induced by spiral waves, the inner disk exerts a positive gravitational torque onto the planet and the outer disk exerts a negative gravitational torque onto the planet. According to analytical analysis, the overall torque is generally negative and, hence, forces the planet to migrate inward. Ward (1997) pointed out that depending on the mass of the protoplanet two major kinds of migration exist. In the so called Type I migration, the planet's mass is small and the response of the disk is linear; the migration speed is very fast so that the migration timescale is as short as $\sim 10^{4} \mathrm{yr}$ for a planetary core of $10 M_{\oplus}$ in a minimum mass solar nebula at $\sim 5.2 \mathrm{AU}$ with sufficient viscosity (see Figure 14 of Ward 1997). In Type II migration, the protoplanet is massive enough to open a gap inside the disk, and migrates on a much longer viscous timescale. Besides classical analytical analysis, many groups have studied the nonlinear evolution of a disk-planet system using numerical multi-dimensional hydrodynamics (Kley 1999; Nelson et al. 2000). These numerical simulations showed that the nonlinear evolution of the orbital migration of a planet inside a disk agrees with linear analysis in a qualitative manner. Masset (2001) and Masset \& Papaloizou (2003) suggested that the corotation torque originated from the coorbital region may play a very different role from that of Lindblad torques; this leads to a third kind of migration referred to as Type III migration, in which the migration happens on a timescale as short as a few tens of orbits and can be directed outward in some cases.

Klahr \& Bodenheimer (2003) described a Baroclinic Instability in non-barotropic disks that may contribute to vorticity and global turbulence, then argued that strong vorticities may contribute to rapid formation of Jupiter-size gas planet (Klahr \& Bodenheimer 2006). Koller et al. (2003) and Li et al. (2005) showed that the so-called RWIs may develop at the local minima of $\mathrm{PV}$, or vortensity (defined as the ratio between local vorticity and surface density), in an inviscid disk with initially uniform PV distribution. Li et al. (2005) showed that non-axisymmetric RWIs lead to the formation of vorticies and density blobs, which exert stronger torque onto the planet when they travel around its vicinity and bring large oscillations to the total torque acted on the planet. They further argued that this mechanism may be possible to change the direction of the migration. Non-axisymmetric RWIs are also relevant to evolution of a single disk (Li et al. 2000) and stellar models with strong differential rotation (Ou \& Tohline 2006). In this work, we study the generation of PV through baroclinic effect and subsequent development of RWIs in systems consisting of a planet and a non-barotropic disk.

\section{Basic Equations, Methods and Initial Setup}

To investigate the interaction between a disk and an embedded planet requires coupling hydrodynamics and orbital dynamics together. Here, we follow Nelson et al. (2000) and many previous investigations to reduce the problem to a two-dimensional (2D) one since the disk thickness is of the order of or smaller than the planetary Hill radius. Three dimensional (3D) investigations will be postponed to future. The fluid motion inside the disk is described by the vertically integrated continuity equation (2.1), radial and 
azimuthal components of the Navier-Stokes equation (2.2) and (2.3),

$$
\begin{aligned}
\frac{\partial \Sigma}{\partial t}+\nabla \cdot(\Sigma \vec{v}) & =0 \\
\frac{\partial\left(\Sigma v_{r}\right)}{\partial t}+\nabla \cdot\left(\Sigma v_{r} \vec{v}\right) & =\frac{\Sigma v_{\phi}^{2}}{r}-\frac{\partial P}{\partial r}-\Sigma \frac{\partial \Phi}{\partial r}+f_{r} \\
\frac{\partial\left(\Sigma v_{\phi}\right)}{\partial t}+\nabla \cdot\left(\Sigma v_{\phi} \vec{v}\right) & =-\frac{\Sigma v_{r} v_{\phi}}{r}-\frac{1}{r} \frac{\partial P}{\partial \phi}-\frac{\Sigma}{r} \frac{\partial \Phi}{\partial \phi}+f_{\phi},
\end{aligned}
$$

where $\Sigma$ is disk surface density, $\vec{v}$ is two fluid velocities, $P$ is vertically integrated pressure, $f_{r}$ and $f_{\phi}$ are two components of viscous forces, and $\Phi$ is the gravitational potential felt by fluid elements. Details regarding viscous terms can be found in Nelson et al. (2000). The EOS of the disk fluid is considered as locally isothermal (Nelson et al. 2000) as given by $P=c_{s}^{2} \Sigma$, where the local isothermal sound speed is $c_{s}=\frac{H}{r} \sqrt{G M_{*} / r}$ with disk aspect ratio $H / r=0.05$. In order to compare with globally isothermal situation, we also carried out one run with a uniform value of $c_{s}=\frac{H}{r} \sqrt{G M_{*} / r_{p}}$, where $r_{p}=1$.

We further simplify our study to non-self-gravitational systems, in which the selfgravity of the fluid is not taken into account for the fluid motion; hence, $\Phi=\Phi_{*}+\Phi_{p}$, where $\Phi_{*}$ is the potential field of the central star and $\Phi_{p}$ is the potential field of the planet, which is given by $\Phi_{p}=-M_{p} / \sqrt{r^{2}+\epsilon^{2}}$, where $M_{p}$ is the planet mass and $\epsilon$ is taken to be 0.2 times the Roche Lobe of the planet. The initial disk model has Keplerian rotational profile and uniform density, which results in an initial radial PV profile $\xi(r)$ that is proportional to $r^{-\frac{3}{2}}$. The value of density and viscosity are chosen to follow those specified in de Val-Borro et al. (2006). To handle the hydrodynamics part, we adopted a legacy code developed by the astrophysical group at Louisiana State University to study star formation (Tohline 1980). The code is explicit and 2nd order in both space and time. It splits the source term and advection term in a manner similar to Zeus (Stone \& Norman 1992). Other features implemented include Van Leer upwind scheme, artificial viscosity to handle shock, and, staggered cylindrical grids. The code is originally threedimensional, but adapted to $2 \mathrm{D}$ in this work. At the boundary of our computational grids, mass is allowed to flow off the grids but no inflow is allowed. We also implemented the wave-killing boundary condition specified in de Val-Borro et al. (2006). The planet is put on a fixed orbit at $r=1 \mathrm{AU}$ in most of our simulations. We also allowed the planet to move in some runs to study the effect of RWIs on its migration. The units adopted are the following: the gravitational constant $G=1$, length unit is $1 \mathrm{AU}$, and $M_{*}+M_{p}=1$. Some brief results follow in the next section.

\section{Simulation results}

We present simulation results for systems with a Neptune-mass planet embedded in either a locally isothermal disk (non-barotropic) or an isothermal disk (barotropic). Fig.1a illustrates a linear color map of surface density distribution on a polar plot for a locally isothermal disk at $t \approx 150$ orbits. Focusing on the formation of high density areas (red/brown regions), we observe not only red Lindblad spiral arms, but also, other nonaxisymmetric high density structures at different locations: the inner edge of the outer disk (brown arc-shaped region centered around 7 o'clock), the outer edge of the inner disk (red/brown arc-shaped region around its edge); interestingly, the density inside the gap is no longer axisymmetric any more, as suggested by the light blue region around 10 oclock. The radial locations of these non-axisymmetric structures match exactly with the local minima of the PV distribution (see Fig.4 of Ou et al. 2007), which is consistent with previous studies on RWIs ( $\mathrm{Li}$ et al. 2000) that RWIs are capable of introducing 

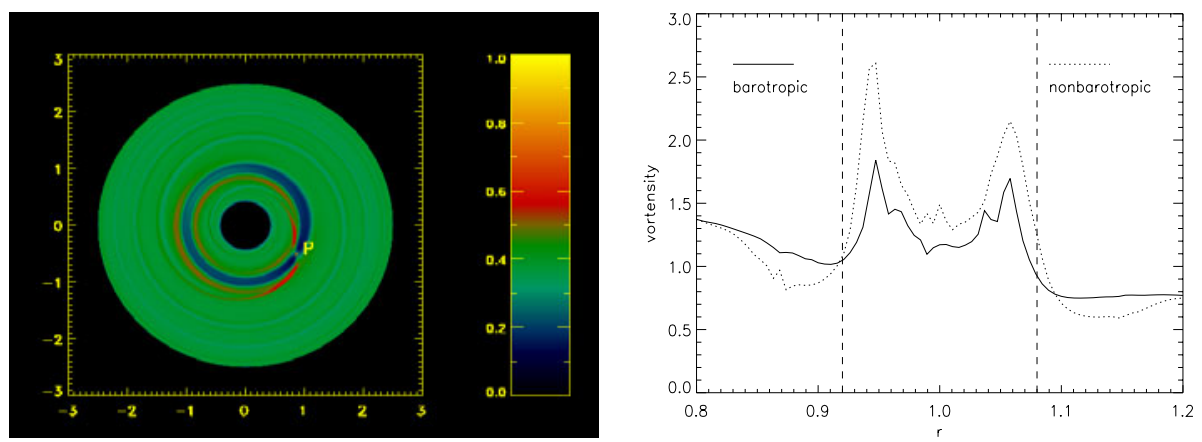

Figure 1. Simulations for an embedded Neptune-mass planet in an inviscid disk. Left panel: (1a) Color map of the disk surface density distribution in linear scale $t \approx 150$ orbits for a resolution run $(400 \mathrm{x} 1600)$. A letter "P" is labeled next to the location of the planet. The color bar represents relative rather than absolute values. Right panel: (1b) Comparison of azimuthally-averaged radial PV profile between two disk-planet systems with a planet embedded in a locally isothermal disk (non-barotropic) and an isothermal disk (barotropic). Vertical dashed lines illustrate edges of the horseshoe region. The planet is located at $r=1$.

non-axisymmetric density distribution in a disk. The total torque acted on the planet is negative in average; however, as time goes on, it varies greatly in amplitude (see $\mathrm{Ou}$ et al. 2007 for more details). This is because those high density blobs formed through RWIs exert a stronger torque on the planet when they are traveling in the vicinity of the planet, hence, large amplitude of oscillations show up in the time evolution of the total torque. Our results on the temporal oscillating behavior of the total torque is consistent with those in Li et al. (2005).

In general, we expect vorticity (hence, PV) to be generated around the shock outside coorbital region, where density gradient and pressure gradient are not aligned to each other. However, detailed analysis shows that PV is generated both near shocks and around the planet in the coorbital region. To understand the mechanism that generates $\mathrm{PV}$ within the coorbital region, we note that our disk models have a non-barotropic EOS with a radial variation of sound speed $c_{s}$, which brings in misalignment of pressure gradient and density gradient wherever azimuthal density gradient appears $\left(\nabla c_{s} \times \nabla \Sigma \neq 0\right)$. This misalignment acts as a source term for the generation of vorticity within the coorbital region, where no shock but strong azimuthal density gradient presents. Therefore, the large density depression in the same region naturally gives birth to PV increment. Fig.1b shows a comparison of azimuthally averaged radial PV profiles for two runs at $t \approx 60$ orbits: the disk in one run has an isothermal EOS (barotropic) and the other disk has a locally isothermal EOS (non-barotropic). The PV peaks in the non-barotropic disk is significantly higher than those of barotropic disk. Furthermore, a peak located around the planet $(r=1)$ shows up in the non-barotropic disk. To further examine if our results are resolution-dependent, we carried out a run with resolution $800 \times 3200$ for $\sim 70$ orbits. The simulation exhibits PV maxima within the coorbital region as well (see $\mathrm{Ou}$ et al. 2007). As a further evidence that PV is generated around where azimuthal density gradient exists, Figure $2 \mathrm{a}$ and $2 \mathrm{~b}$ illustrate the distribution of $\mathrm{PV}$ increment and azimuthal density gradient, respectively, for the run with resolution $800 \times 3200$ at $t \sim 70$ orbits. For a better view, they are zoomed in the neighborhood of the planet. It is observed that $\mathrm{PV}$ is generated within the Roche lobe, where strong azimuthal density gradient, instead of shocks, exists. Such a generating mechanism of PV inside a planet's Roche lobe has the same origin as baroclinic instability discussed in Klahr \& Bodenheimer (2003), which contributes to global turbulence within the disk. On the other hand, we also observe that 

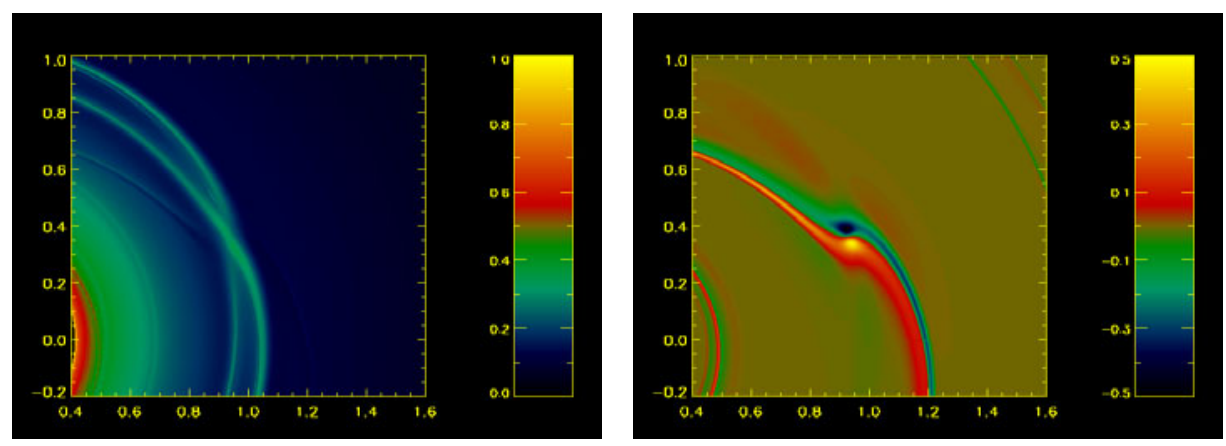

Figure 2. Simulations for the Neptune-mass planet in disk. Left panel: (2a)Distribution of PV around the Neptune-mass planet at $t=70$ orbits for the run with resolution $800 \times 3200$. Shocks are well resolved. Vortensity is generated within the coorbital region, especially in the Roche lobe, where no shock exists. The azimuthal density gradient within the same region acts as a source term of PV generation. Right panel: (2b) Distribution of azimuthal density gradient at the same time. Strong density gradient is observed within the Roche lobe and coorbital region.

the oscillations of the torque acted on a Neptune-mass planet appear much earlier in our simulations compared to previous studies, which suggests that PV generation is even more effective and common in non-barotropic disk models (see also Ou et al. 2007).

As shown in Figure 2b, the azimuthal density gradient flips the sign across the planet; thus, we expect the PV increment also flip the sign there. However, the PV change within the Roche-lobe shown in Figure 2a is always positive, this is possibly due to PV mixture along librating stream lines in the horse region where fluid elements make the U-turn. A detailed and rigorous analysis of fluid dynamics and PV mixture around a Neptune-mass planet embedded in a non-barotropic disk will be carried out in a separate investigation.

In our simulations of a freely moving Neptune-mass planet (Ou et al. 2007), RWIs do not change the overall picture of inward migration of a Neptunian planet; but they have significant influence on the torque exerted on the planet and make the migration speed of the planet non-monotonic.

\section{Summary and Discussion}

In this work, we carried out high resolution simulations on the interaction between a protoplanetary disk and an embedded planet with emphasis on the interplay between a disk and a planet under the influence of baroclinic generation of PV. Our results are consistent with classical analysis on the interaction between a protoplanetary disk and an embedded planet through Lindblad torques. We confirmed previous outcomes that non-axisymmetric RWI is likely to develop under certain circumstances and have an important influence on the migration of a planet inside an inviscid disk. We also found that the generation of $\mathrm{PV}$ is more common and effective in disks with non-barotropic EOS through the baroclinic instability, further favoring the development of RWIs. As the asymmetry of the density distribution induced by RWIs becomes prominent, the resulting density blobs exert periodical and enhanced gravitational pull onto the planet as they pass by the vicinity of the planet, which causes the total torque received by the planet undergo large amplitude oscillations.

Our analysis shows that strong vorticity has been generated around the planet through baroclinic effect, this may help its core to accrete materials faster in a way suggested in Klahr \& Bodenheimer (2006) and shorten the time scale needed to form a Jupiter mass planet. As a side effect of an inwardly migrating planet, RWIs introduce 
non-axisymmetric density blobs along the way. These enhanced density blobs with strong vortices may help rapid formation of new planet cores within them, especially in inner regions of circumstellar disks where rapid precipitation and coagulation of solid materials are likely to happen (Silverstone 2006). If these new-born cores could survive during the migration of a giant planet (Raymond 2006), they may produce Earth-like planets in the Habitable Zones (Ji et al. 2007) or Hot Earths interior to a close-in giant planet (Raymond 2006). One important issue is where this non-barotropic mechanism is expected to occur in a protoplanetary disk. Typically, at small radii $(r<10 \mathrm{AU})$, the disk flow can not dissipate its internal energy in a time less than the horseshoe U-turn time, the locally isothermal approximation does not hold there. Therefore, we expect this mechanism is likely to happen at larger radii. On the other hand, the breakdown of the locally isothermal approximation does not necessarily remove the temperature variation. If temperature variation preserves at smaller radii, it may still favor baroclinic effect. In realistic situations, this could be a much more complicated process than what is described here. Further investigations on these issues with full 3D simulations and disk self-gravity (see also Baruteau \& Masset 2008; Zhang \& Zhou 2008 in this issue) are under way.

\section{Acknowledgements}

We thank the anonymous referee for useful comments and suggestions that helped to improve the contents. J.H.J. acknowledges the financial support by the National Natural Science Foundations of China (Grants 10573040, 10673006, 10203005, 10233020) and the Foundation of Minor Planets of Purple Mountain Observatory. S.O. was partially supported by NSF grant AST-0407070.

\section{References}

Baruteau, C. \& Masset, F. 2008, IAU S249, this issue

Goldreich, P., Tremaine, S., 1978, ApJ, 222, 850

Ida, S., \& Lin, D.N.C. 2004, ApJ, 604, 388

Ji, J., Kinoshita, H., Liu, L., \& Li, G.Y. 2007, ApJ, 657, 1092

Klahr, H.H., Bodenheimer, P., 2003, ApJ, 582, 869

Klahr, H.H., Bodenheimer, P., 2006, ApJ, 639, 432

Kley, W. 1999, MNRAS, 303, 696

Koller, J., Li, H., Lin, D.N.C., 2003, ApJ, 596, 91

Li, H., et al., 2000, ApJ, 533, 1023

Li, H., et al. 2005, ApJ, 624, 1003

Lissauer, J. J. 1993, ARAA, 31, 129

Masset, F.S., 2001, ApJ, 558, 453

Masset, F.S., Papaloizou, J.C.B., 2003, ApJ, 588, 494

Nelson, R.P., Papaloizou, J.C.B., Masset, F., Kley, W., 2000, MNRAS, 318, 18

Ou, S., Tohline, J.E., 2006, ApJ, 651, 1068

Pollack, J. B., et al. 1996, Icarus, 124, 62

Raymond, S. N., Mandell, A. M., \& Sigurdsson, S. 2006, Science, 313, 1413

Safronov, V.S. 1969, Evolution of the Protoplanetary Cloud and Formation of the Earth and the Planets (Moscow:Nauka)

Silverstone, M.D., et al., 2006, ApJ, 639, 1138

Stone, J.M., Norman, M.L., 1992, ApJS, 80, 753

Tohline, J. E. 1980, ApJ, 235, 866

de Val-borro, M., et al. 2006, MNRAS, 370, 529

Ward, W. R. 1997, Icarus, 126, 261

Zhang, H., Zhou, J.-L. 2008, IAU symposium 249: Exoplanets: Detection, Formation and Dynamics, eds. Y.-S. Sun, S. Ferraz-Mello \& J.-L. Zhou, p. 413 